\title{
Association of lipoprotein(a) and major adverse cardiovascular events in patients with percutaneous coronary intervention
}

\author{
Zhihao Chen ${ }^{1}$, Chaohui Jiang ${ }^{1}$, Huimin Qu ${ }^{1}$, Shuang Liang ${ }^{1}$, Jian Yang ${ }^{2}$, Hui Wu², Chao He², \\ Xinan Wang ${ }^{2}$
}

\begin{abstract}
${ }^{1}$ Department of Cardiology, Xiang'an Hospital of Xiamen University, Xiamen, China ${ }^{2}$ Department of Cardiology, The First College of Clinical Medical Sciences, China Three Gorges University, Yichang, Hubei, China
\end{abstract}

Submitted: 11 August 2018

Accepted: 8 September 2018

Arch Med Sci 2019; 15 (6): 1375-1380

DOI: https://doi.org/10.5114/aoms.2018.79401

Copyright (c) 2018 Termedia \& Banach

\begin{abstract}
Introduction: The aim of the current study was to evaluate the association between lipoprotein(a) [Lp(a)] and major adverse cardiovascular events (MACEs) in patients with percutaneous coronary intervention ( $\mathrm{PCI}$ ) treatment. Material and methods: This was a retrospective study. The demographics, prior medical histories, comorbidities and laboratory parameters were collected from the electronic health record. All participants were followed up for 1 year after the indexed $\mathrm{PCl}$. Studied end points were a composite of MACEs including all-cause mortality, non-fatal myocardial infarction (MI), non-fatal ischemic stroke, transient ischemic attack and stent restenosis.

Results: During 1-year follow-up, 87 MACEs occurred. Compared to patients who did not have MACEs, patients who had MACEs were older, more likely to have higher body mass index, diabetes mellitus and left main lesion, and also had higher baseline low density lipoprotein cholesterol (LDL-C) and Lp(a) levels. All patients in both groups were prescribed aspirin and clopidogrel at discharge. Nearly $97.4 \%$ and $95.4 \%$ of patients in both groups were treated with statins and a higher proportion of patients in the MACE group were treated with ezetimibe $(11.5 \%$ vs. $3.5 \%, p<0.05)$. In multivariate regression analysis, diabetes mellitus, LDL-C, Lp(a) and glomerular filtration rate were independent risk factors for MACEs; statin use appeared to be a protective factor for MACEs. Patients with increased Lp(a) level had significantly higher incidence of MACEs than the normal $L p(a)$ level group $(p=0.001)$.
\end{abstract}

Conclusions: Baseline serum LP(a) can be used to predict MACEs in patients after PCI treatment, which was independent of LDL-C.

Key words: lipoprotein(a), major adverse cardiovascular events, percutaneous coronary intervention.

\section{Introduction}

Low density lipoprotein cholesterol (LDL-C) is a major risk factor for atherosclerotic cardiovascular diseases (ASCVD) [1-4]. Furthermore, a substantial number of clinical studies have demonstrated that lower serum level of LDL-C is associated with better cardiovascular prognosis [5-7]. Besides LDL-C, in the last two decades, some studies have shown that lipoprotein(a) [Lp(a)] might be an independent risk factor for ASCVD [8-10]. However, some studies suggested that after accounting for the effects of

\author{
Corresponding author: \\ Zhihao Chen \\ Department of Cardiology \\ Xiang'an Hospital \\ of Xiamen University \\ 2000 Xiang'an Donglu \\ Xiang'an District \\ 361000 Xiamen, China \\ Phone: +86 0592-2181220 \\ E-mail:chenzhihao@tom.com
}


LDL-C, the association between $\operatorname{Lp}(\mathrm{a})$ and cardiovascular outcome was insignificant [11-14].

Structurally [15], Lp(a) comprises two essential components, namely LDL-C and apolipoprotein(a) [Apo(a)]. Contrary to LDL-C, Lp(a) catabolism is less dependent on LDL-C receptor, and prior studies have shown that statins have a modest effect on Lp(a) regulation [15]. Pathophysiologically [16], compared to LDL-C, Lp(a) is not only capable of promoting atherosclerosis but also capable of enhancing thrombus formation through impairing fibrinolysis. The pathophysiological process of coronary artery stent restenosis is characterized by endothelial dysfunction, smooth muscle cell proliferation and fibrin accumulation [17]. Therefore, with respect to the unique features of $L p(a)$ in terms of pro-atherosclerosis and pro-thrombosis, one may anticipate that increased serum Lp(a) level could be associated with higher risk of cardiovascular events. However, the data on the association between serum Lp(a) level and cardiovascular outcomes including coronary artery stent restenosis in Chinese populations are limited $[18,19]$.

Therefore, we conducted a retrospective cohort study to evaluate the association between baseline serum Lp(a) level and cardiovascular outcomes in patients within 1 year after percutaneous coronary intervention ( $\mathrm{PCl}$ ) treatment. We believe that data from our current study will provide insights into the function of $L p(a)$ as regards cardiovascular outcomes in patients after coronary artery stenting. In addition, our current study also will provide a foundation for clinical trials aimed at reducing cardiovascular events in the future.

\section{Material and methods}

\section{Participants' enrollment}

Our current study was approved by the Clinical Research Ethic Committee of Xiang'an Hospital of Xiamen University and the First College of Clinical Medical Sciences of China Three Gorges University. Since this was a retrospective study, no informed consent was required from participants. The inclusion criteria were as follows: $\geq 18$ years old, received $\mathrm{PCl}$ at our hospital and had follow-up at our hospital within the first year after PCI treatment. The exclusion criteria were as follows: had documented familial dyslipidemia, and had severe complications including hemodynamic instability, acute coronary artery stent thrombosis or coronary artery dissection during indexed $\mathrm{PCl}$. The study schematic diagram is presented in Figure 1.

\section{Data collection}

The demographics such as age and gender were collected from the electronic health record (EHR). Body mass index (BMI) was calculated as weight in kilograms divided by height in squared meters. Prior documented medical histories and comorbidities were collected from the EHR. Laboratory parameters were also collected from the EHR. Per hospital protocol, fasting blood samples were drawn before the indexed $\mathrm{PCl}$. Serum level of $L p(a)$ was measured with a sandwich enzyme linked immune-sorbent assays (ELISA kit, Yaji Biosystems, Shanghai, China). All the procedures were performed in accordance with the manual instructions and were assessed by a SYNCHRON LX20 UniCel DxC800 analyzer (Beckman Coulter Inc., USA). Baseline serum creatinine level was used to calculate the glomerular filtration rate (GFR) in accordance with the Modification of Diet in Renal Disease (MDRD) formula [20].

\section{Studied end points}

All participants were followed up for 1 year after the indexed $\mathrm{PCl}$ and parameters of interest were collected from the EHR. In specific, studied end points of the current study were the composite of major adverse cardiovascular events (MACEs) including all-cause mortality, non-fatal myocardial infarction (MI), non-fatal ischemic stroke, transient ischemic attack and stent restenosis. All the events were adjudicated by independent cardiologists. All data extraction was conducted by two investigators.

\section{Statistical analysis}

Continuous data were presented as mean \pm standard deviation (SD) or median (interquartile

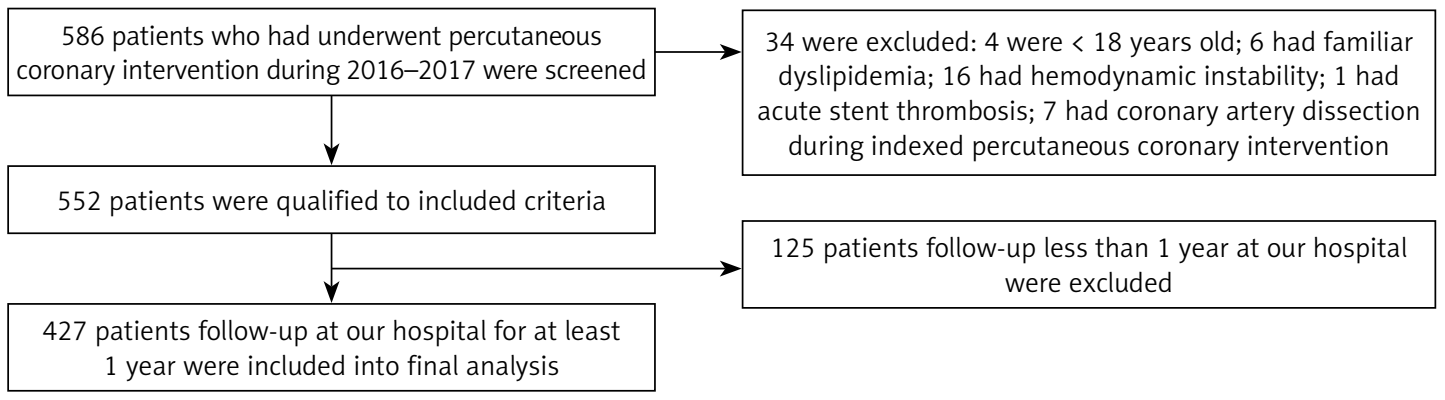

Figure 1 . Study schematic diagram 
range, IQR) as appropriate, and were compared by Student's $t$-test when data were normally distributed, and otherwise were compared by the Wilcoxon rank-sum test. Categorical data were presented as number (proportion) and compared by the $\chi^{2}$ test or Fisher's exact test as appropriate. Univariate and multivariate regression analysis was performed to evaluate the association between serum Lp(a) level and MACEs. A Kaplan-Meier survival curve was plotted to evaluate the accumulative incidence of MACEs between normal and increased serum $L p(a)$ level groups, and the cutoff value was $30 \mathrm{mg} / \mathrm{dl}$ in accordance with prior reports $[11,15]$. All reported $p$-values were 2 -sided, and a $p$-value of $<0.05$ was considered statistically significant. All statistical analyses were conducted with the SPSS statistical package for Windows version 19.0 (SPSS Inc., Chicago, Illinois).

\section{Results}

\section{Participants' enrollment}

Participants' enrollment was performed from January of 2016 to January of 2017. As presented in Figure 1, a total of 586 patients who underwent $\mathrm{PCl}$ in our hospital during this period were screened and 34 were excluded in accordance with the exclusion criteria. Among the 552 patients, 427 who were followed up at our hospital for at least 1 year after the indexed PCI were recruited into the final analysis.

\section{MACEs during 1-year follow-up}

During the first year follow-up, a total of 87 (20.4\%) MACEs occurred. In specific, 3 (3.4\%) patients died, 19 (21.8\%) patients had non-fatal MI, $22(26.3 \%)$ patients had non-fatal ischemic stroke, $9(10.3 \%)$ had transient ischemic attack and 34 (39.1\%) had stent restenosis. All the events were adjudicated by independent cardiologists. Per study protocol, all patients were divided into two groups, namely without and with MACEs groups, and between-group differences were evaluated.

\section{Baseline characteristics}

As presented in Table I, compared to patients who did not have MACEs, patients who had MACEs during the follow-up were older $(57.9 \pm 12.2$ years vs. $52.2 \pm 13.8$ years), and were more likely to have higher BMI $\left(25.1 \pm 5.0 \mathrm{~kg} / \mathrm{m}^{2}\right.$ vs. $23.4 \pm 4.2$ $\mathrm{kg} / \mathrm{m}^{2}$ ), diabetes mellitus (37.9\% vs. $32.1 \%$ ) and left main lesion (20.7\% vs. $14.1 \%)$, and also had higher baseline LDL-C (3.5 $\pm 0.6 \mathrm{mmol} / \mathrm{l}$ vs. $3.0 \pm 0.8$ $\mathrm{mmol} / \mathrm{l})$ and $\mathrm{Lp}(\mathrm{a})(55.6 \pm 14.3 \mathrm{mg} / \mathrm{dl}$ vs. $29.8 \pm 7.6$ $\mathrm{mg} / \mathrm{dl}$ ) levels ( $p<0.05$ for all comparisons). No other significant differences were observed.

\section{Medications used at discharge}

As presented in Table II, all patients in both groups were prescribed aspirin and clopidogrel at discharge.

Table I. Baseline characteristic comparisons

\begin{tabular}{|c|c|c|}
\hline Variables & $\begin{array}{l}\text { Without } \\
\text { MACEs } \\
(n=340)\end{array}$ & $\begin{array}{l}\text { MACEs } \\
(n=87)\end{array}$ \\
\hline Male, $n(\%)$ & $196(57.6)$ & $48(55.2)$ \\
\hline Age [years] & $52.2 \pm 13.8$ & $57.9 \pm 12.2^{*}$ \\
\hline Current smoker, $n$ (\%) & $86(25.3)$ & $25(28.7)$ \\
\hline Body mass index $\left[\mathrm{kg} / \mathrm{m}^{2}\right]$ & $23.4 \pm 4.2$ & $25.1 \pm 5.0^{\star}$ \\
\hline Hypertension, $n$ (\%) & $144(42.4)$ & $37(44.8)$ \\
\hline Diabetes mellitus, $n(\%)$ & $109(32.1)$ & $33(37.9)^{\star}$ \\
\hline Dyslipidemia, n (\%) & $128(37.6)$ & $35(40.2)$ \\
\hline Atrial fibrillation, $n$ (\%) & $16(4.7)$ & $6(6.9)$ \\
\hline Ischemic stroke, $n$ (\%) & $40(11.8)$ & $10(11.5)$ \\
\hline $\begin{array}{l}\text { Peripheral vascular } \\
\text { disease, } n(\%)\end{array}$ & $28(8.2)$ & $8(9.2)$ \\
\hline Chronic heart failure, $n(\%)$ & $22(6.5)$ & $7(8.0)$ \\
\hline $\begin{array}{l}\text { Systolic blood pressure } \\
{[\mathrm{mm} \mathrm{Hg}]}\end{array}$ & $129 \pm 18$ & $131 \pm 20$ \\
\hline $\begin{array}{l}\text { Diastolic blood pressure } \\
{[\mathrm{mm} \mathrm{Hg}]}\end{array}$ & $70 \pm 16$ & $72 \pm 19$ \\
\hline Heart rate [beats/min] & $78 \pm 16$ & $79 \pm 14$ \\
\hline Total cholesterol $[\mathrm{mmol} / \mathrm{l}]$ & $4.5 \pm 0.7$ & $4.8 \pm 0.7$ \\
\hline Triglyceride [mmol/l] & $1.6 \pm 0.6$ & $1.7 \pm 0.8$ \\
\hline LDL-C [mmol/l] & $3.0 \pm 0.8$ & $3.5 \pm 0.6^{*}$ \\
\hline $\mathrm{HDL}-\mathrm{C}[\mathrm{mmol} / \mathrm{l}]$ & $1.1 \pm 0.4$ & $1.0 \pm 0.3$ \\
\hline Lipoprotein(a) [mg/dl]) & $29.8 \pm 7.6$ & $55.6 \pm 14.3^{*}$ \\
\hline Creatinine $[\mu \mathrm{mol} / \mathrm{l}]$ & $70.4 \pm 15.2$ & $71.9 \pm 13.8$ \\
\hline $\begin{array}{l}\text { Glomerular filtration rate } \\
{\left[\mathrm{ml} / \mathrm{min} / 1.73 \mathrm{~m}^{2}\right]}\end{array}$ & $81.9 \pm 10.4$ & $78.3 \pm 9.5$ \\
\hline $\begin{array}{l}\text { Fasting plasma glucose } \\
{[\mathrm{mmol} / \mathrm{l}]}\end{array}$ & $5.8 \pm 0.7$ & $5.7 \pm 0.9$ \\
\hline Hemoglobin $A_{1 c}(\%)$ & $6.3 \pm 0.6$ & $6.4 \pm 0.5$ \\
\hline Left main, $n(\%)$ & $48(14.1)$ & $18(20.7)^{\star}$ \\
\hline $\begin{array}{l}\text { Left anterior descending, } \\
n(\%)\end{array}$ & $117(34.4)$ & $32(36.8)$ \\
\hline Left circumflex, $n(\%)$ & $86(25.3)$ & $23(26.4)$ \\
\hline Right coronary artery, $n(\%)$ & $103(30.3)$ & $28(32.1)$ \\
\hline Number of stents implanted & $1.5 \pm 0.7$ & $1.6 \pm 0.8$ \\
\hline Drug-eluting stents, $n$ (\%) & $335(95.5)$ & $82(94.3)$ \\
\hline $\begin{array}{l}\text { Left ventricular ejection } \\
\text { fraction (\%) }\end{array}$ & $52.4 \pm 8.6$ & $50.7 \pm 7.9$ \\
\hline
\end{tabular}

${ }^{*} P<0.05$ versus without MACE group; MACE - major adverse cardiovascular events, $L D L-C$ - low-density lipoprotein cholesterol, $H D L-C-$ high-density lipoprotein cholesterol. 
Table II. Medication use at discharge

\begin{tabular}{|c|c|c|}
\hline Medications & $\begin{array}{l}\text { Without } \\
\text { MACEs } \\
(n=340)\end{array}$ & $\begin{array}{l}\text { MACEs } \\
(n=87)\end{array}$ \\
\hline Aspirin, $n(\%)$ & $340(100)$ & $87(100)$ \\
\hline Clopidogrel, $n$ (\%) & $340(100)$ & $87(100)$ \\
\hline Statins, $n(\%)$ & $331(97.4)$ & $83(95.4)$ \\
\hline Ezetimibe, $n(\%)$ & $12(3.5)$ & $10(11.5)^{\star}$ \\
\hline ACEi/ARB, $n(\%)$ & $302(88.8)$ & $76(87.4)$ \\
\hline$\beta$-Blocker, $n(\%)$ & $286(84.1)$ & $73(83.9)$ \\
\hline Oral anti-coagulation, $n(\%)$ & $4(1.2)$ & $2(2.3)$ \\
\hline $\begin{array}{l}\text { Calcium channel blocker, } \\
n(\%)\end{array}$ & $83(24.4)$ & $20(23.0)$ \\
\hline Diuretic, $n(\%)$ & $20(5.9)$ & $5(5.7)$ \\
\hline $\begin{array}{l}\text { Oral anti-diabetic } \\
\text { medication, } n(\%)\end{array}$ & $65(19.1)$ & $18(20.7)$ \\
\hline Insulin, $n(\%)$ & $33(9.7)$ & $10(11.5)$ \\
\hline
\end{tabular}

Nearly $97.4 \%$ and $95.4 \%$ of patients in both groups were treated with statins and a higher proportion of patients in the MACE group were treated with ezetimibe than the group without MACE $(11.5 \%$ vs. $3.5 \%$, $p<0.05)$. No significant differences in use of other medications were observed.

\section{Independent risk factors for MACEs}

Univariate and multivariate regression analysis were performed to evaluate the independent risk factors for MACEs. As presented in Table III, in the univariate regression analysis, age, current smoking, BMI, diabetes mellitus, LDL-C, Lp(a), GFR and statin use were all independently associated with MACEs. In the multivariate regression analysis, only diabetes mellitus (hazard ratio $(H R)=$ 1.12 and $95 \%$ confidence interval (Cl): 1.04-1.42), LDL-C (HR = 1.08 and 95\% Cl: 1.04-1.32), Lp(a) $(\mathrm{HR}=1.05$ and $95 \% \mathrm{Cl}: 1.02-1.24)$ and GFR $(\mathrm{HR}=1.22$ and $95 \% \mathrm{Cl}: 1.12-1.67)$ were independent risk factors for MACEs, while statin use (HR = 0.97 and $95 \% \mathrm{Cl}$ : 0.92-0.99) appeared to be a protective factor for MACEs.

\section{Accumulative incidence of MACEs by different $\operatorname{Lp}(\mathrm{a})$ level}

Based on prior reports [10, 15], serum level of $\mathrm{Lp}(\mathrm{a})<30 \mathrm{mg} / \mathrm{dl}$ was considered as the normal range and accumulative incidence of MACEs was compared between normal and increased Lp(a) level groups. As presented in Figure 2, patients with an increased $L p(a)$ level had significantly higher incidence of MACEs during the first year's follow-up than that in the normal $L p(a)$ level group $(p=0.001)$.

\section{Discussion}

Dyslipidemia, characterized by increased serum LDL-C level, is a major risk factor for ASCVD [21]. Prior randomized clinical trials have demonstrated that a reduced LDL-C level with statins is beneficial to prevent cardiovascular events. However, some studies have shown that despite LDL-C level being within the target range, some patients still have recurrent cardiovascular events. It is speculated that other cholesterol components

Table III. Independent risk factors for MACEs

\begin{tabular}{|lcc|}
\hline Variables & $\begin{array}{c}\text { Univariate } \\
\text { HR (95\% Cl) }\end{array}$ & $\begin{array}{c}\text { Multivariate } \\
\text { HR (95\% Cl) }\end{array}$ \\
\hline Age [years] & $1.20(1.09-1.57)$ & $1.08(0.96-1.15)$ \\
\hline Male gender (vs. No) & $1.04(0.96-1.12)$ & NS \\
\hline Current smoking (vs. No) & $1.15(1.04-1.32)$ & $1.02(0.92-1.23)$ \\
\hline Body mass index [kg/m²] & $1.20(1.09-1.38)$ & $1.04(0.94-1.17)$ \\
\hline Hypertension (vs. No) & $1.11(0.92-1.25)$ & NS \\
\hline Diabetes mellitus (vs. No) & $1.34(1.16-1.86)$ & $1.12(1.04-1.42)$ \\
\hline Chronic heart failure (vs. No) & $1.05(0.95-1.19)$ & NS \\
\hline LDL-C [mmol/l] & $1.28(1.14-1.61)$ & $1.08(1.04-1.32)$ \\
\hline Lipoprotein(a) [mg/dl] & $1.24(1.11-1.53)$ & $1.05(1.02-1.24)$ \\
\hline Left main (vs. No) & $1.09(0.90-1.45)$ & NS \\
\hline Left ventricular ejection fraction (\%) & $1.15(0.97-1.06)$ & NS \\
\hline Glomerular filtration rate [ml/min/1.73 $\left.\mathrm{m}^{2}\right]$ & $1.40(1.28-1.94)$ & $1.22(1.12-1.67)$ \\
\hline Statins (vs. No) & $0.92(0.89-0.97)$ & $0.97(0.92-0.99)$ \\
\hline Ezetimibe (vs. No) & $0.97(0.90-1.03)$ & $\mathrm{NS}$ \\
\hline
\end{tabular}

MACE - major adverse cardiovascular events, $L D L-C$ - low-density lipoprotein cholesterol, $H R$ - hazard ratio, $C l$ - confidence interval, NS - non-significant. 
such as Lp(a) may be associated with residual cardiovascular risk [22]. However, prior findings are conflicting and the data on the Chinese populations are limited. Our current study shows that in the Chinese patients after $\mathrm{PCl}$ treatment, independent of LDL-C, baseline serum Lp(a) level is useful to predict 1-year cardiovascular outcomes and increased serum Lp(a) level portends a higher MACE rate than among those with a normal Lp(a) level.

Of note, a substantial number of patients after $\mathrm{PCl}$ treatment still experience recurrent cardiovascular events despite serum LDL-C level being within the target range. The underlying mechanisms are multifactorial. Prior studies have shown that increased $L p(a)$ level might contribute to the residual cardiovascular risk. Indeed, experimental studies suggested that $L p(a)$ induced endothelial dysfunction, inflammatory reaction and oxidative stress, which in turn led to atherosclerotic initiation and development. Furthermore, some clinical studies, but not all, have also shown that increased $L p(a)$ level was associated with higher cardiovascular risk. Consistent with prior reports, our current study also showed that among patients after $\mathrm{PCl}$ treatment, those who had MACEs during follow-up had higher baseline serum LDL-C and $L p(a)$ levels. After adjusting for potential confounding factors including LDL-C, LP(a) remained independently associated with MACEs, indicating that $L p(a)$ was useful to predict MACEs in the Chinese patients after $\mathrm{PCl}$ treatment. However, since our study was a retrospective study, a causal relationship could not be confirmed and future studies are warranted to corroborate our current findings.

Interestingly and importantly, our current study showed that the percentage of stent restenosis was highest compared to other cardiovascular events including all-cause mortality, non-fatal MI, non-fatal ischemic stroke and transient ischemic attack. Since all participants were followed up at our hospital and all participants were compliant with dual antiplatelet therapy during the first year of follow-up after $\mathrm{PCl}$ treatment, we considered that the higher stent restenosis risk might not be attributable to noncompliance with dual antiplatelet treatment. In contrast, it might be associated with higher serum Lp(a) level. Indeed, authors $[23,24]$ reported that in Chinese patients after $\mathrm{PCl}$ treatment, baseline increased LDL-C and Lp(a) portended a higher risk of revascularization. Consistent with their study, our current study also found that those with increased Lp(a) level had higher stent restenosis risk. However, in the prior study, after adjusting for baseline LDL-C level, the association between $L p(a)$ and risk of coronary revascularization was insignificant. In our current study, we found that even after adjusting for potential covariates including LDL-C, increased serum L $\mathrm{p}(\mathrm{a})$

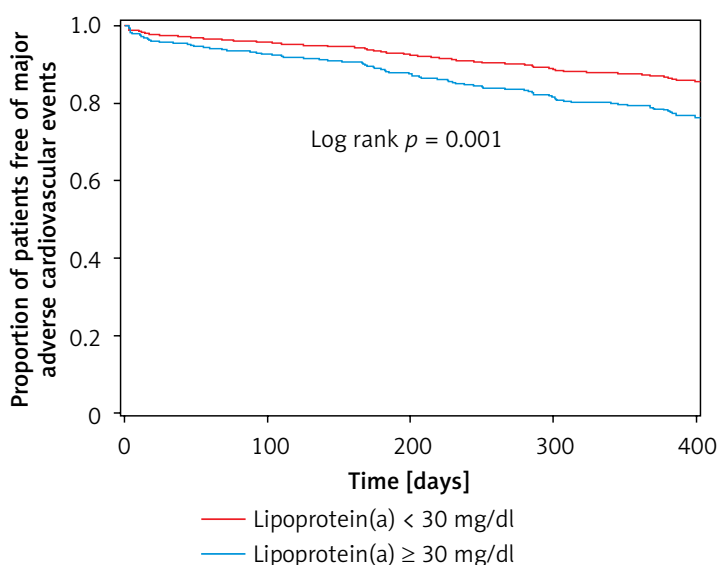

Figure 2. Survival curve

level at baseline remained significantly associated with stent restenosis, with a hazard ratio of 1.16 (95\% Cl: 1.04-1.25, $p=0.013$ ). Unfortunately, although our study and other studies have shown the association between Lp(a) and cardiovascular events including stent restenosis, no effective and efficient approach could be used to reduce $L p(a)$ level up till now.

With respect to our preliminary findings, future directions should be focused on corroborating our findings from the national level of China as well as in other population groups. Basic research should be conducted to evaluate the underlying mechanisms. In addition, future randomized, prospective, large-scale trials are necessary to demonstrate whether reducing the $L p(a)$ level can prevent cardiovascular events in patients with an achieved LDL-C target after treatment $[23,24]$.

Some limitations of our current study should be mentioned: first of all, this was a retrospective study which was subjected to the limitation of temporal relationship in terms of the association between exposure and outcomes; second, the observational design could not allow us to infer a causal relationship; third, although we adjusted for potential confounding factors, unmeasured and undetected confounding factors still existed which could have impacted the results; fourth, our current study concerned a retrospective cohort and no LDL-C was obtained after finishing follow-up. However, our current study was focused on the baseline LDL-C and Lp(a) levels in relation to cardiovascular outcomes. In addition, in the regression analysis model, we adjusted for baseline LDL-C level. In the future prospective cohort study, it is necessary to evaluate LDL-C change during follow-up; last but not the least, our current study was conducted in a Chinese population and therefore future studies performed in other racial/ethnic populations are warranted to corroborate our findings.

In conclusion, our current study shows that in Chinese patients with $\mathrm{PCl}$ treatment, baseline 
serum $L p(a)$ level can be used to predict MACEs, especially stent restenosis, and all these findings are independent of baseline LDL-C level. Future randomized trials are necessary to demonstrate whether reducing the $L p(a)$ level can further prevent cardiovascular events in patients with an achieved LDL-C target.

\section{Acknowledgments}

We greatly appreciate the help that Dr. Jie Chen provided to us in performing statistical analysis.

\section{Conflict of interest}

The authors declare no conflict of interest.

\section{References}

1. Ference BA, Ginsberg HN, Graham I, et al. Low-density lipoproteins cause atherosclerotic cardiovascular disease. 1. Evidence from genetic, epidemiologic, and clinical studies. A consensus statement from the European Atherosclerosis Society Consensus Panel. Eur Heart 2017; 38: 2459-72.

2. Silverman MG, Ference BA, Im K, et al. Association between lowering LDL-C and cardiovascular risk reduction among different therapeutic interventions: a systematic review and meta-analysis. JAMA 2016; 316: 1289-97.

3. Howard BV, Robbins DC, Sievers ML, et al. LDL cholesterol as a strong predictor of coronary heart disease in diabetic individuals with insulin resistance and low LDL: the Strong Heart Study. Arterioscler Thromb Vasc Biol 2000; 20: 830-5.

4. Soran H, Adam S, Mohammad JB, et al. Hypercholesterolaemia - practical information for non-specialists. Arch Med Sci 2018; 14: 1-21.

5. Storey BC, Staplin N, Haynes R, et al. Lowering LDL cholesterol reduces cardiovascular risk independently of presence of inflammation. Kidney Int 2018; 93: 1000-7.

6. Wadhera RK, Steen DL, Khan I, Giugliano RP, Foody JM. A review of low-density lipoprotein cholesterol, treatment strategies, and its impact on cardiovascular disease morbidity and mortality. J Clin Lipidol 2016; 10: 472-89.

7. Rodriguez F, Harrington RA. Cholesterol, cardiovascular risk, statins, PCSK9 inhibitors, and the future of LDL-C lowering. JAMA 2016; 316: 1967-8.

8. Sun L, Zong M, Chen C, et al. Low LPA gene kringle IV-2 repeat copy number association with elevated lipoprotein (a) concentration as an independent risk factor of coronary atherosclerotic heart disease in the Chinese Han population. Lipids Health Dis 2018; 17: 111.

9. Erqou S, Kaptoge S, Perry PL, et al. Lipoprotein(a) concentration and the risk of coronary heart disease, stroke, and nonvascular mortality. JAMA 2009; 302: 412-23.

10. Cai A, Li L, Zhang Y, Mo Y, Mai W, Zhou Y. Lipoprotein(a): a promising marker for residual cardiovascular risk assessment. Dis Markers 2013; 35: 551-9.

11. Cai A, Li L, Zhang Y, et al. Baseline LDL-C and Lp(a) elevations portend a high risk of coronary revascularization in patients after stent placement. Dis Markers 2013; 35: 857-62.

12. LuC G, Bard JM, Arveiler D, et al. Lipoprotein (a) as a predictor of coronary heart disease: the PRIME Study. Atherosclerosis 2002; 163: 377-84.
13. Suk DJ, Rifai N, Buring JE, Ridker PM. Lipoprotein(a), measured with an assay independent of apolipoprotein(a) isoform size, and risk of future cardiovascular events among initially healthy women. JAMA 2006; 296: 1363-70.

14. Cantin B, Gagnon F, Moorjani S, et al. Is lipoprotein(a) an independent risk factor for ischemic heart disease in men? The Quebec Cardiovascular Study. J Am Coll Cardiol 1998; 31: 519-25.

15. Nordestgaard BG, Chapman MJ, Ray K, et al. Lipoprotein(a) as a cardiovascular risk factor: current status. Eur Heart J 2010; 31: 2844-53.

16. Saeed A, Virani SS. Lipoprotein(a) and cardiovascular disease: current state and future directions for an enigmatic lipoprotein. Front Biosci 2018; 23: 1099-112.

17. Buccheri D, Piraino D, Andolina G, Cortese B. Understanding and managing in-stent restenosis: a review of clinical data, from pathogenesis to treatment. J Thorac Dis 2016; 8: E1150-62.

18. Dai W, Long J, Cheng Y, Chen Y, Zhao S. Elevated plasma lipoprotein(a) levels were associated with increased risk of cardiovascular events in Chinese patients with stable coronary artery disease. Sci Rep 2018; 8: 7726.

19. Cai DP, He YM, Yang XJ, Zhao X, Xu HF. Lipoprotein (a) is a risk factor for coronary artery disease in Chinese Han ethnic population modified by some traditional risk factors: a cross-sectional study of 3462 cases and 6125 controls. Clin Chim Acta 2015; 451: 278-86.

20. K/DOQI clinical practice guidelines for chronic kidney disease: evaluation, classification, and stratification. Am J Kidney Dis 2002; 39 (2 Suppl 1): S1-266.

21. Banach M, Jankowski P, Jóźwiak J, et al. PoLA/CFPiP/PCS guidelines for the management of dyslipidaemias for family physicians 2016. Arch Med Sci 2017; 13: 1-45.

22. Sahebkar A, Simental-Mendía LE, Watts GF, Serban MC, Banach M. Comparison of the effects of fibrates versus statins on plasma lipoprotein(a) concentrations: a systematic review and meta-analysis of head-to-head randomized controlled trials. BMC Med 2017; 15: 22.

23. Kotani K, Banach M. Lipoprotein(a) and inhibitors of proprotein convertase subtilisin/kexin type 9. J Thorac Dis 2017; 9: E78-82.

24. Awad K, Mikhailidis DP, Katsiki N, Muntner P, Banach M. Effect of ezetimibe monotherapy on plasma lipoprotein(a) concentrations in patients with primary hypercholesterolemia: a systematic review and meta-analysis of randomized controlled trials. Drugs 2018; 78: 453-62. 